\title{
Previsão de Poluentes Atmosféricos Utilizando Modelos Estatísticos de Regressão na Região Metropolitana do Recife
}

\author{
Prediction of Atmospheric Pollutants Using Statistical Models in the Metropolitan Region \\ of Recife
}

Daniel Campos ${ }^{1}$ (D) orcid.org/0000-0002-9329-6220

\author{
Manoel Marinho' ${ }^{1}$ (D) orcid.org/0000-0003-3129-0453 \\ ${ }^{1}$ Escola Politécnica de Pernambuco, Universidade de Pernambuco, Recife, Brasil. \\ E-mail do autor principal: Daniel Campos danielsilvacampos@hotmail.com
}

\begin{abstract}
RESUMO
O Material Particulado (PM) é um poluente atmosférico resultante da queima de combustíveis e que fica suspenso no ar na forma de poeira, fumaça, etc. A exposição prolongada a este material pode, em alguns casos, ocasionar mortes. O presente artigo tem a finalidade de encontrar a melhor curva de regressão múltipla para previsão da concentração horária do Material Particulado com diâmetro inferior a $10 \mu \mathrm{m}$ (PM10). Inicialmente utilizou-se apenas a concentração do PM10 em até 6 horas anteriores como variável explanatória das equações de regressão (M1), em seguida, foram incluídas as variáveis meteorológicas (M2): velocidade do vento, umidade relativa do ar e temperatura. Através de uma busca exaustiva de 63 combinações, foi possível estimar a concentração de poluente exclusivo em função do PM10. Assim como, estimou-se mais de 15 milhões de combinações entre os elementos meteorológicos com o objetivo de realizar melhoria na previsão efetuada por essa abordagem. $O$ coeficiente de determinação (R2) para o ajuste do modelo $M 2(R 2=0,64)$ possui valor superior que 0 $M 1(R 2=0,54)$. Esse fato demonstra que a inclusão dos parâmetros físicos meteorológicos resulta em melhoria na capacidade explanatória realizada no modelo de regressão. Ambos os modelos apresentaram resultados satisfatórios, sobressaindo-se o modelo M2.
\end{abstract}

Palavras-Chave: Poluentes Atmosféricos; Material Particulado; Série temporal; Regressão Linear.

\section{ABSTRACT}

Particulate Material (PM) is an air pollutant resulting from the relative burn of fuels, this pollutant stay suspended in the air in the dust form, smoke, etc. The prolonged exposition to this material results in deaths in some cases. The present article has the goal to find the better multiple regression curve in order to forecast hourly concentration of diameter less than $10 \mu \mathrm{m}$ particulate material (PM10). At first, only six hours delay of particulate material concentration were used as explanatory variable on the regression equations (M1), in the following, have been included the meteorological variables (M2): wind speed, relative humidity in the air and air temperature. By computational exhaustive search from 63 combinations, it was possible to estimate PM10 pollutant concentration as function of himself. Furthermore, was estimated more than 15 million combinations between meteorological elements in order to explore improvements in the predictions provided by the last approach. The coefficient of determination (R2) for the adjustment of the model $M 2(R 2=0,64)$ is bigger than $M 1(R 2=0,54)$, demonstrating that inclusion of meteorological physical parameters results in improvements on the explanatory capacity of the regression model. Both models afford good results, standing out the M2 model.

KEY-WORDS: Atmospheric Pollutants; Particulate Material; Temporal series; Linear Regression. 


\section{INTRODUÇÃO}

Ao longo das últimas décadas tem-se assistido o auge da intervenção humana sobre o planeta, principalmente após o surgimento das indústrias siderúrgicas, produtos químicos e a queima de combustíveis fósseis. Essas intervenções não foram acompanhadas de análises que possibilitassem avaliar o impacto causado ao meio ambiente ou os prováveis danos à saúde. Portanto, há alguns anos é possível observar as consequências prejudiciais causadas por este processo desordenado. O objetivo atual possui como finalidade adquirir conhecimento sobre os resíduos resultantes de modo a evitar os efeitos prejudiciais ao ambiente e consequentemente, aos seus habitantes.

$\mathrm{O}$ ar apresenta-se como elemento com maior prejuízo devido à intervenção humana no ambiente. Este fato ocorre principalmente nos grandes centros urbanos, que possuem como principais fontes poluidoras a crescente presença de automóveis juntamente com a indústria [1].

A poluição atmosférica refere-se à contaminação do ar pela introdução de substâncias conhecidas por poluentes atmosféricos, os mesmos podem se apresentar em forma de gases e partículas provenientes de fontes naturais (vulcões, neblinas) ou produzidas pela atividade humana (queima de carvão, indústrias, escapamento de veículos). A poluição atmosférica traz prejuízos não somente à saúde e à qualidade de vida das pessoas, mas também acarretam maiores gastos do Estado, decorrentes do aumento do número de atendimentos e internações hospitalares, além do uso de medicamentos, custos esses que poderiam ser evitados com a melhoria da qualidade do ar nos centros urbanos. Existe, igualmente, o prejuízo causado à qualidade dos materiais (corrosão), do solo e das águas (chuvas ácidas) além de afetar a visibilidade. Ademais, a poluição do ar é considerada pela OMS (Organização Mundial da Saúde) o maior risco ambiental para a saúde no mundo, e a estimativa é a de que todos os anos cerca de 8 (oito) milhões de pessoas morrem em decorrência desta exposição $[2, \mathbf{3}]$.

Entre os poluentes atmosféricos que mais acarretam em preocupações à saúde pública, estão incluídos: o material particulado (PM), o monóxido de carbono (CO), o ozônio (O3) e o dióxido de nitrogênio (NO2) [3]. Dentre os poluentes citados, aquele que causa um maior impacto negativo à saúde humana é o PM. Este, normalmente é medido pela concentração de partículas finas suspensas no ar com diâmetro menor que 10,0 micrômetros (PM10) ou 2,5 micrômetros (PM2,5). Essas partículas são capazes de penetrar profundamente no sistema respiratório e causar danos à saúde $[4,5]$.

A maior parcela do material particulado urbano é originada pela queima de combustível, por processos industriais, pela névoa salina e ressuspensão do solo. Sua dispersão ocorre em função das condições meteorológicas e da topografia local. Enquanto que os efeitos adversos à saúde humana dependem de suas propriedades físico-químicas, diâmetro médio e tempo de exposição. O principal efeito é irritação no sistema respiratório, devido principalmente, ao PM10. Este material particulado transforma quimicamente os poluentes iniciais em substâncias mais nocivas, e atua como vetor de microrganismos, substâncias orgânicas e cancerígenas [6].

Estimativas das concentrações de PM10 permitem avaliar os padrões de qualidade do ar e possíveis ações de proteção à população, particularmente em eventos extremos. O uso da modelagem matemática computacional fornece condições de quantificar as tendências temporais de cada poluente atmosférico e chegar a uma estimativa dos potenciais efeitos à saúde com relação a poluição do ar. Portanto, o estudo da qualidade do ar, assim como a simulação para previsão acurada de seus principais poluentes, se torna de grande valia para fins de prevenção e controle, sendo a principal importância deste tema creditada à relação existente entre as altas concentrações de poluentes, e os efeitos adversos à saúde humana $[4,7,8]$.

Estudos prévios utilizaram modelos estatísticos e métodos de inteligência computacional para analisar e prever as concentrações de material particulado e outros poluentes presentes no ar. Alguns trabalhos estudaram abordagens variadas, como a regressão linear, não linear e métodos de aprendizado de máquinas para avaliar qual deles fornecem uma previsão mais acurada da concentração do PM10 em região urbana [8]. Arquiteturas de rede neural do tipo MLP, com otimização de parâmetros por meio de algoritmo genético e ajuste de fase foi desenvolvida e testada na previsão tanto do PM10 quanto do PM2.5 $[9,4]$. Estudos que comparam a capacidade de previsão 
do modelo de regressão linear múltipla com outros modelos estatísticos, como o autorregressivo e de Box-Jenkis, para séries temporais de poluentes atmosféricos, também já foi trabalhado [10]. Assim como análises envolvendo aplicação de máquinas desorganizadas (Extreme Learning Machines - ELM) e rede neural com estado de eco (Echo State Networks - ESN) na previsão do PM [11].

Os objetivos deste trabalho foram de estimar a concentração do poluente atmosférico PM10 em função das condições meteorológicas utilizando modelos estatísticos de regressão linear e avaliar a influência dos parâmetros meteorológicos na concentração deste mesmo poluente na Região Metropolitana do Recife (RMR) - PE.

\section{MATERIAIS E MÉTODOS}

\subsection{PM10 e Variáveis Meteorológicas}

Foram obtidas concentrações de material particulado com diâmetro inferior a 10 $\mu \mathrm{m}$ (PM10, $\left.\mu \mathrm{g} / \mathrm{m}^{3}\right)$, presentes no ar, para o período de 17/07/2015 a 09/04/2017, através da Agência Estadual de Meio Ambiente de Pernambuco $(\mathrm{CPRH})$. Para o mesmo período do poluente atmosférico, foram obtidas as medidas meteorológicas: velocidade do vento (WS, $\mathrm{m} / \mathrm{s}$ ), umidade relativa do ar $(\mathrm{RH}, \%)$ e temperatura (AT, $\left.\mathrm{C}^{\circ}\right)$. Todas as medições foram feitas em intervalos de $1 \mathrm{~h}$ e foram extraídas da estação de monitoramento do ar instalada na Escola de Referência em Ensino Médio Frei Otto, em Nossa Senhora do Ó, localizada no município de Ipojuca, Estado de Pernambuco. O sistema da estação de monitoramento capta as informações em tempo real por meio de sensores e envia todo o conteúdo pela internet para a sede da agência na cidade do Recife-PE.

A Figura 1 mostra a localização aproximada do ponto de coleta (Estação Gaibú, acima a direita) cujas coordenadas geográficas são: $8^{\circ} 19^{\prime} 44,37^{\prime \prime}$ (S) e $34^{\circ} 57^{\prime} 22,66^{\prime \prime}$ (O). Na parte inferior esquerda, com o círculo amarelo, se encontra a Refinaria Abreu e Lima (RNEST) na rodovia PE 60.

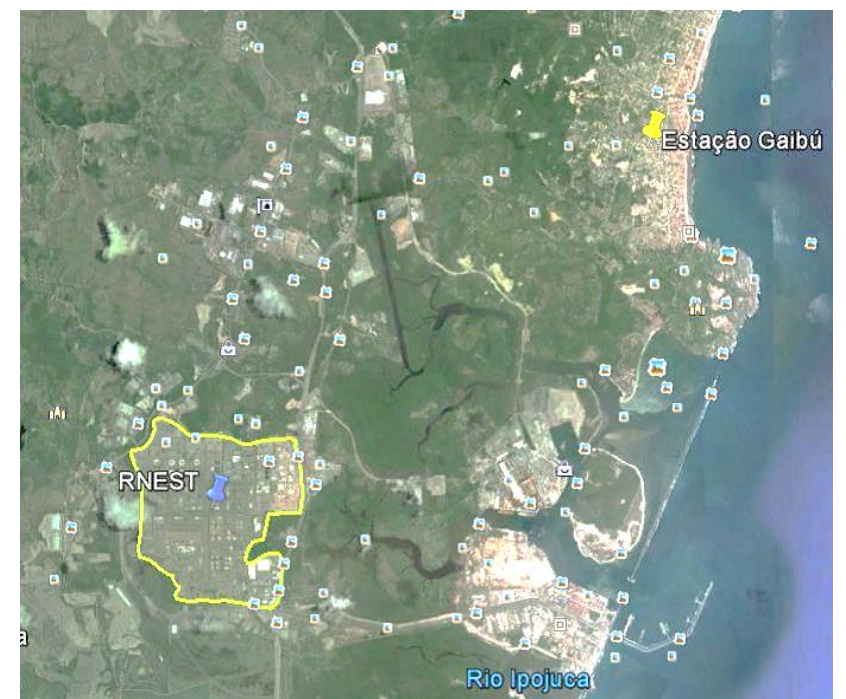

Figura 1: Localização aproximada da Estação de Qualidade do Ar.

Fonte: JCTM, Monitoramento da Qualidade do $\mathrm{Ar}$, Estação RNEST - GAIBU, 26/02/2017 a 25/03/2017.

A Tabela-1 apresenta a estatística descritiva para as séries de dados referentes ao PM10 e parâmetros meteorológicos usados neste estudo.

Tabela 1: Estatística dos dados utilizados.

\begin{tabular}{|lccc|}
\hline Parâmetro & Média & $\begin{array}{l}\text { Desvio } \\
\text { Padrão }\end{array}$ & $\begin{array}{l}\text { Coeficiente de } \\
\text { Variação }\end{array}$ \\
\hline $\begin{array}{l}\text { PM10 } \\
\left(\mu \mathrm{g} / \mathrm{m}^{3}\right)\end{array}$ & 35,802 & 22,998 & 0,648 \\
\hline $\mathrm{WS}(\mathrm{m} / \mathrm{s})$ & 3,422 & 0,957 & 0,290 \\
\hline RH $(\%)$ & 73,507 & 9,139 & 0,121 \\
\hline AT $\left({ }^{\circ} \mathrm{C}\right)$ & 27,762 & 2,110 & 0,072 \\
\hline
\end{tabular}

Fonte: Autor.

\subsection{Modelos de Regressão Linear}

A análise de regressão é uma técnica estatística para modelar e investigar a relação entre duas ou mais variáveis, muitos problemas em engenharia e ciências envolvem explorar essa relação [12]. Esse tipo de relacionamento é representado por um modelo matemático, que, na verdade, se trata de uma equação que associa a variável dependente (que se deseja prever) às variáveis independentes (preditoras). Sendo assim, análise de regressão tem por objetivo desvendar o comportamento entre uma variável dependente e as consideradas independentes.

Para fins de aplicação dos modelos de regressão linear, foram desenvolvidos dois modelos em 
linguagem C, denominados de RLS (Regressão Linear Simples) e RLM (Regressão Linear Múltipla). Estes, ao receberem as séries de dados apropriadas, se encarregam de efetuar as operações de regressão. Foi utilizado o ambiente de desenvolvimento integrado (IDE) de código aberto Code::Blocks (www.codeblocks.org/) versão 16.01, para codificar, compilar e executar os programas. Ambos foram implementados contendo um algoritmo de busca exaustiva em sua estrutura com a finalidade de gerar todas as possíveis combinações de variáveis independentes e verificar com qual delas obtêm-se as melhores previsões para os poluentes considerados. A variável dependente é representada por PM10, as variáveis independentes são: WS-i, RH-i e AT-i, além de PM10-i (em que "-i" representa a série de dados defasada em i hora(s)).

O modelo RLS explora a relação entre duas variáveis, para este caso, considera-se uma única variável independente (preditora, $x$ ) e uma única variável dependente (ou variável de resposta, Y) a formulação do modelo de regressão linear simples é descrita a seguir:

$$
Y=\beta_{0}+\beta_{1} x,(1)
$$

em que $\beta_{0}$ e $\beta_{1}$ são coeficientes de regressão desconhecidos a princípio. Para obter os coeficientes, o modelo RLS utiliza estimativa de mínimos quadrados, estimando a interseção $\left(\beta_{0}\right)$ e a inclinação da reta $\left(\beta_{1}\right)$ por:

$$
\beta_{0}=\dot{y}+\beta_{1} \dot{x}
$$

e

$$
\beta_{1}=\frac{a}{b},(3)
$$

em que:

$$
\begin{gathered}
a=\sum_{i=1}^{n} y_{i} x_{i}-\frac{\left(\sum_{i=1}^{n} y_{i}\right)\left(\sum_{i=1}^{n} x_{i}\right)}{n}, \\
b=\sum_{i=1}^{n} x_{i}^{2}-\frac{\left(\sum_{i=1}^{n} x_{i}\right)^{2}}{n}, \\
\dot{y}=\frac{1}{2} \sum_{i=1}^{n} y_{i}
\end{gathered}
$$

$$
\dot{x}=\frac{1}{2} \sum_{i=1}^{n} x_{i},
$$

e nrepresenta o número de pares de observação $\left(x_{1}, y_{1}\right),\left(x_{2}, y_{2}\right), \ldots,\left(x_{n}, y_{n}\right)$.

Aplicações mais interessantes da análise de regressão geralmente exigem o uso de mais de uma variável regressora, pois muitas vezes, uma única variável não é capaz de explicar tudo a respeito da variável resposta. Para tanto, o modelo RLM analisa a relação entre duas ou mais variáveis, caracterizando assim modelos de regressão linear múltipla. Nesta abordagem, a variável resposta Y pode estar relacionada com duas ou mais variáveis independentes, sendo sua formulação descrita a seguir:

$$
Y=\beta_{0}+\beta_{1} x_{1}+\beta_{2} x_{2}+\cdots+\beta_{k} x_{k},(4)
$$

em que, $\beta_{0}$ representa a interseção do plano, todos os parâmetros $\beta_{j,} j=1,2,3, \ldots, k$, são chamados de coeficientes de regressão e a estimativa destes parâmetros pode ser feita através do método dos mínimos quadrados. Agora, as observações da série são descritas da seguinte forma, $n$ continua representando o número de observações e suponha que $n>k$, seja $x_{i j}$ a i-ésima observação, logo as observações são:

$$
\left(x_{i 1}, x_{i 2}, x_{i 3}, \ldots, x_{i k}, y_{i}\right), i=1,2,3, \ldots, n ; n>k \text {, }
$$

em que cada uma das observações satisfaz a equação (4) [12].

O modelo RLM realiza a estimativa dos coeficientes de regressão através da resolução do sistema de equações normais de mínimos quadrados (5).

$$
\left\{\begin{array}{c}
n \beta_{0}+\beta_{1} \sum_{i=1}^{n} x_{i 1}+\beta_{2} \sum_{i=1}^{n} x_{i 2}+\cdots+\beta_{k} \sum_{i=1}^{n} x_{i k}=\sum_{i=1}^{n} y_{i} \\
\beta_{0} \sum_{i=1}^{n} x_{i 1}+\beta_{1} \sum_{i=1}^{n} x_{i 1}^{2}+\beta_{2} \sum_{i=1}^{n} x_{i 1} x_{i 2}+\cdots+\beta_{k} \sum_{i=1}^{n} x_{i 1} x_{i k}=\sum_{i=1}^{n} x_{i 1} y_{i} \\
\vdots \\
\beta_{0} \sum_{i=1}^{n} x_{i k}+\beta_{1} \sum_{i=1}^{n} x_{i k} x_{i 1}+\beta_{2} \sum_{i=1}^{n} x_{i k} x_{i 2}+\cdots+\beta_{k} \sum_{i=1}^{n} x_{i k}^{2}=\sum_{i=1}^{n} x_{i k} y_{i} .
\end{array}\right.
$$

A resolução do sistema é efetuada utilizando-se o algoritmo de eliminação gaussiana (ou método de escalonamento), que simplifica um sistema de 
várias incógnitas e o resolve automaticamente, fornecendo ao usuário os $k$ coeficientes de regressão de cada equação, para cada combinação de variáveis.

\subsection{Métricas de Desempenho}

Para medir o desempenho de cada equação de regressão ao problema proposto, os dois modelos foram desenvolvidos para trabalhar com 2 métricas principais, são elas: erro quadrado médio (mean square error - MSE) e o índice de concordância (index agreement - IA). Além do Coeficiente de Determinação $\left(R^{2}\right)$ e o Coeficiente de Correlação (R).

O $R^{2}$ varia entre 0 e 1 , indicando em percentagem o quanto o modelo consegue explicar a variação dos valores observados para o PM10. 0 $\mathrm{R}$ varia entre -1 e 1 , servindo para medir o grau com que duas variáveis estão correlacionadas e a direção dessa correlação (se positiva ou negativa). É importante salientar que, o índice de concordância, é utilizado para indicar o nível de concordância entre a equação de regressão e a variável prevista, sendo a principal métrica adotada neste trabalho por conta de sua constante utilização em outros estudos sobre o tema.

O MSE é frequentemente utilizado na previsão de séries temporais, sua principal função é indicar se a previsão feita pelo modelo está superestimada ou subestimada.

No entanto, não se pode tomar conclusões apenas com base no MSE $[13,14]$. Quanto mais próximo de zero, melhor os resultados de previsão fornecidos pelo modelo testado.

$$
M S E=\frac{1}{2} \sum_{i=1}^{n}\left(Y-y_{i}\right)^{2},(6)
$$

em que $Y$ representa o valor previsto pelo modelo e $y_{i}$ o valor observado (real) na série da variável resposta.

O IA foi desenvolvido como uma medida padronizada do grau de erro de previsão do modelo e varia entre 0 e 1 . Um valor de 1 para o IA indica uma correspondência (concordância) perfeita entre o modelo e a variável prevista, e 0 indica nenhum tipo de concordância entre eles [15].

$$
I A=1-\frac{\sum_{i=1}^{n} Y-y_{i} \vee \quad 2}{\sum_{i=1}^{n}}
$$

em que ý representa a média de todas as observações da variável de saída.

No desenvolvimento dos modelos de regressão foi levado em consideração uma defasagem de até 6 (seis) horas para as variáveis independentes. As equações de regressão trabalhadas utilizam até 3 (três) elementos meteorológicos e sempre no mínimo uma série de dados defasadas do poluente como variável independente. Sendo assim, contabiliza-se 4 (quatro elementos) $\times 6$ (defasagem de seis horas para cada variável) $=24$ (vinte e quatro) variáveis no total a serem consideradas no desenvolvimento das equações de regressão.

Usando o conceito matemático de combinação linear, é possível calcular que foram geradas 15.600.443 (mais de 15 milhões de combinações) equações de regressão no total, sendo que, cada uma delas fornece um resultado diferente da previsão, melhor ou pior, por isso a importância da implementação de um algoritmo de busca exaustiva que teste cada uma delas e indique com qual obteve-se a melhor métrica IA $e$, consequentemente as melhores métricas.

$\mathrm{Na}$ fase de treino, foram utilizados os dados de concentração do $\mathrm{PM}$ a partir das $0 \mathrm{~h}$ do dia 17/07/2015 até as $22 \mathrm{~h}$ do dia 08/02/2017, totalizando 13.751 horas. Os dados de $23 \mathrm{~h}$ do dia 08/02/2017 até as $23 \mathrm{~h}$ do dia 09/04/2017 (num total de 1.441 horas) foram direcionadas para testar cada uma das equações obtidas na fase de treino.

\section{RESULTADOS E DISCUSSÃO}

Foram analisados os resultados fornecidos pelos dois modelos (RLS e RLM). Primeiramente, os resultados obtidos quando se utiliza o PM10 para prever a si mesmo sem levar em consideração os parâmetros meteorológicos, a posteriori, os resultados referentes às equações que levam em consideração, além do próprio PM10 defasado, também os parâmetros meteorológicos. Foi dada especial atenção as equações que obtiveram o melhor desempenho na previsão da concentração do poluente em estudo.

\subsection{Modelo de Previsão sem Parâmetros Meteorológicos}


A Tabela-2 apresenta o Coeficiente de Determinação $\left(R^{2}\right)$, o Coeficiente de Correlação $(R)$, além das métricas já discutidas, entre o PM10 e os dois melhores modelos de previsão obtidos. É analisado inicialmente o modelo que não leva em consideração as variáveis meteorológicas (1). Utilizando o PM10 defasado em até 6 horas como variável independente e usando todas as combinações possíveis destas, foram obtidas 63 equações de regressão.

Observa-se que, a relação linear entre o PM10 e sua concentração na hora anterior apresenta um $\mathrm{R}^{2}=0,54$, revelando que as concentrações do PM10 podem ser influenciadas fortemente por seus valores registrados em horas anteriores. O IA fornecido pela equação foi próximo a 1 , indicando uma boa correspondência (concordância) entre o modelo 1 e a variável PM10, o MSE é de aproximadamente 124,79 , o menor dentre as demais, indicando que esta equação é a que apresenta uma melhor qualidade em suas previsões, não superestimando ou subestimando

\subsection{Modelo de Previsão com Parâmetros Meteorológicos}

É de particular interesse o estudo da influência das condições climáticas sobre o nível de poluição presente no ar. Os parâmetros meteorológicos, por si só, não oferecem resultados suficientemente aceitáveis na estimativa do PM10, no entanto, fisicamente, é perceptível que a concentração do poluente sofre influência da ação do vento, humidade relativa do ar e temperatura. Portanto, na composição de modelos de regressão, é importante considerar também as variáveis climáticas como dado de entrada da equação.

Foram geradas e testadas 15.600 .380 (além das já citadas no tópico 3.1) equações de regressão múltipla, sendo essas resultantes das combinações obtidas pela defasagem de até 6 horas das variáveis WS, RH, AT e do próprio PM10. A Tabela-2 fornece também o modelo 2 , no qual a melhor equação de regressão que leva em consideração as variáveis meteorológicas na

Tabela 2: Modelos de previsão (1) e (2) e métricas obtidas

\begin{tabular}{|c|c|c|c|c|}
\hline Equação de Regressão & $\mathbf{R}^{\mathbf{2}}$ & $\mathbf{R}$ & IA & MSE \\
\hline (1) $\mathrm{PM} 10=0,733726 *(\mathrm{PM}-1)+9,737346$ & 0,538353 & 0,733725 & 0,863461 & 124,704589 \\
\hline $\begin{array}{c}\text { (2) } \mathrm{PM} 10=-6,711668+(-0,786904) *(\text { AT- } 6)+ \\
(-3,124209) *(\text { AT }-2)+(4,312524) *(\text { AT }-1)+ \\
(1,073921) *(\text { WS }-3)+(0,572355) *(\text { WS }-2)+ \\
(-0,011735) *(\mathrm{PM}-5)+(0,015271) *(\mathrm{PM}-4)+ \\
(0,046042) *(\mathrm{PM}-3)+(0,092772) *(\mathrm{PM}-2)+ \\
(0,581973) *(\mathrm{PM}-1)\end{array}$ & 0,643302 & 0,802061 & 0,886135 & 112,466688 \\
\hline
\end{tabular}

Fonte: Autor.

tanto quanto as outras em seus resultados, demonstrando então a importância de se utilizar o valor da concentração do poluente na hora anterior como variável de entrada para fins de previsão.

Ainda, corroborando com os resultados obtidos, observa-se no modelo 1 que o coeficiente de correlação (R) apresentou um valor de 0,73, indicando uma correlação forte e positiva entre a concentração do PM10 na hora anterior e a atual, confirmando que uma equação de regressão simples pode fornecer resultados razoáveis levando-se em conta que a concentração do material particulado está sendo estimada com base apenas na concentração observada na hora anterior. previsão do PM10 é representada.

Dentre as variáveis atmosféricas consideradas, a umidade relativa foi a que apresentou, em módulo, a maior correlação com o PM10 ( $R=$ $0,54)$, no entanto, de maneira inversa, quando esta aumenta, a concentração do poluente tende a diminuir. Mas, apesar deste resultado, destaca-se a ausência do parâmetro meteorológico RH que não participa como variável de entrada do modelo de regressão 2, na verdade o que se pôde observar foi que, ao se utilizar o parâmetro RH como variável explicativa, as equações seguem a tendência de diminuir seu respectivo IA. Neste ponto, é importante ressaltar que, em geral, quando o tempo está mais seco, ou seja, quando a umidade relativa do ar diminui (no inverno, por exemplo), 
tende a ocorrer um aumento das crises devido a doenças respiratórias que, por sua vez, estão fortemente relacionadas com a elevação da concentração do material particulado e de outros poluentes presentes na atmosfera das grandes cidades.

Quando comparados os dois modelos, uma significativa melhora é percebida no modelo 2, principalmente devido a adição dos parâmetros meteorológicos junto com PM10 em horas anteriores como variável de entrada. Ainda, ocorreu uma diminuição do MSE, demonstrando que, utilizando esta abordagem, se torna possível realizar previsões ainda melhores.

Também, ao comparar os coeficientes de correlação, percebe-se que ocorreu um aumento do potencial explicativo com o uso da equação 2 , comprovando que existe um alto grau de relacionamento linear entre o conjunto de variáveis climáticas consideradas no modelo e o PM10, o que de imediato demonstra a vantagem de se utilizar os parâmetros meteorológicos na previsão do poluente e comprova o relacionamento físico existente entre eles. O coeficiente de determinação também é maior, o que o classifica como um modelo que explica melhor a variação do PM10.

A Figura-2 apresenta um gráfico que representa a concentração real do PM10 (linha preta sólida) e as estimativas feitas pelas equações de regressão 1 (pontilhado) e 2 (linha cinza), para os dias 10 e 11/02/2017.

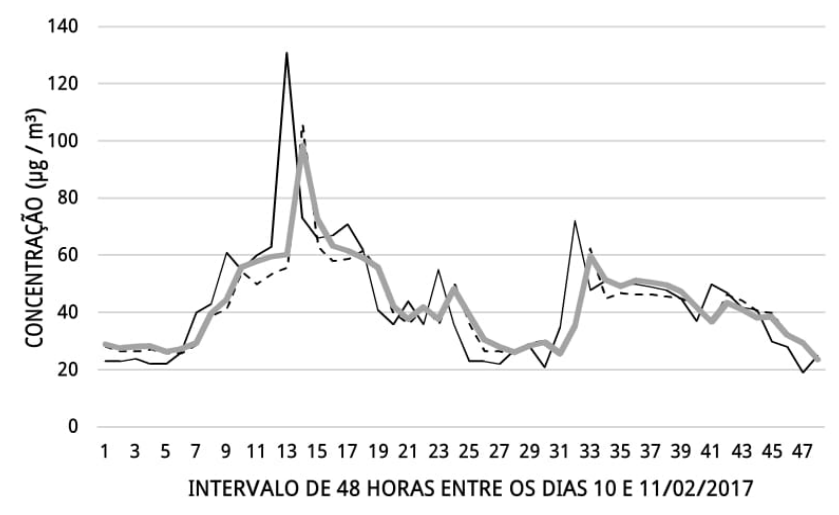

Figura 2: Sobreposição das concentrações do PM10 (em $\mu \mathrm{g} / \mathrm{m}^{3}$ ) real e previsto.

Ambas as equações forneceram bons resultados na maior parte das horas analisadas, no entanto, o gráfico da equação de regressão 2 demonstrou uma aderência maior aos dados de concentração real para algumas horas do dia. Este comportamento ficou evidenciado ao longo do período de tempo considerado.

Por tanto, para as variáveis trabalhadas e a região em estudo, considerou-se que o modelo 2 obteve um melhor desempenho. No entanto, vale mencionar que, a modelagem 2 inevitavelmente requer uma maior quantidade de dados a serem processados pelo computador em uso, isto significando que, apesar desta fornecer melhores previsões, a mesma apresenta uma complexidade computacional notadamente maior quando na busca do modelo regressor que melhor se ajuste a saída desejada, resultando em um tempo mais elevado de processamento.

Para fins de comparação, considerando o tamanho da série histórica trabalhada, o modelo 1 conclui todas as suas combinações em no máximo alguns minutos, enquanto que a modelagem 2 exige alguns poucos dias de busca intermitente a fim de fornecer todos os resultados. Porém, este custo é relativo apenas a fase de busca e, uma vez que os resultados ideais são apresentados, é possível adotá-los e se ocupar em trabalhar apenas com as variáveis que levaram a estes.

\section{CONCLUSÕES}

Apesar da simplicidade do modelo Estatístico de Regressão Linear frente aos métodos mais sofisticados de inteligência computacional para previsões de séries temporais, é possível perceber que ele é capaz de fornecer bons resultados, sendo que a confiabilidade destes é altamente dependente da qualidade dos dados disponíveis e das características físicas das variáveis em estudo. O uso das variáveis meteorológicas temperatura e velocidade do vento, acrescentadas a equação de regressão 2 , permite uma melhoria na estimativa da concentração do poluente PM10, isto pode ser evidenciado através das métricas consideradas e acontece porque existe uma interação real ocorrendo entre as partículas de PM10 que pairam no ar atmosférico e as recorrentes mudanças climatológicas da região metropolitana do Recife (alterações na velocidade dos ventos, variação de temperatura, etc.). Dentre os três parâmetros atmosféricos analisados, a umidade relativa do ar é o que está mais correlacionado com a concentração do poluente, seu aumento, porém, não contribui para a elevação da concentração do PM10. Assim, temperaturas altas e ventos mais intensos contribuem para um clima mais seco que, aliado a presença do material particulado, aumenta 
a incidência de doenças tornando mais grave o quadro dos indivíduos mais vulneráveis a problemas cardiorrespiratórios nas grandes cidades.

\section{AGRADECIMENTOS}

A Agência Estadual de Meio Ambiente (CPRH) pelo fornecimento dos dados necessários. Ao Departamento de Pós-graduação em Engenharia de Sistemas (PPGES) da Escola Politécnica da Universidade de Pernambuco (POLI-UPE), que permitiu as simulações matemáticas em seu laboratório. A Coordenação Setorial de Pósgraduação e Pesquisa da POLI-UPE pela concessão de bolsa de incentivo à pesquisa.

\section{REFERÊNCIAS}

[1] BRAGA, A. et al. Poluição atmosférica e saúde humana. REVISTA USP, n. 51, p. 58-71, 2001.

[2] QUALIDADE DO AR. In: Ministério do Meio Ambiente. Disponível em: http://www.mma.gov. $\mathrm{br} /$ cidadessustentaveis/qualidade-do-ar. Acesso em: 10 jul. 2017.

[3] JASAREVIC, T.; LINDMEIER, C. World Health Assembly closes, passing resolutions on air pollution and epilepsy. In: World Health Organization Media Centre, News release. 26 maio 2015. Disponível em: http://www.who.int/mediacentre/news/releases/2 015/wha-26-may-2015/en/. Acesso em: 26 jul. 2017.

[4] MATTOS NETO, P. S. G. de et al. Hybrid intelligent system for air quality forecasting using phase adjustment. Engineering Applications of Artificial Intelligence: The International Journal of intelligent Real-Time Automation. v. 32, p. 185 - 191, jun. 2014.

[5] The International Bank for Reconstruction and Development / The World Bank. MDGs and the Environment: Agenda for Inclusive and Sustainable Development, Global Monitoring Report. Washington DC, 2008. Technical Report.

[6] LYRA, G. B.; ODA-SOUZA, M.; VIOLA, D. N. Modelos Lineares aplicados à estimativa da concentração do material particulado (PM10) na cidade do Rio de Janeiro, RJ. Revista Brasileira de Meteorologia, v. 26, n. 3, p. $392-400$, 2011.
[7] ZHANG, Ping et al. Temporal and Spatial Simulation of Atmospheric Pollutant PM2.5 Changes and Risk Assessment of Population Exposure to Pollution Using Optimization Algorithms of the Back Propagation-Artificial Neural Network Model and GIS. International Journal of Environmental Research and Public Health, v. 12, n. 10, p. $12171-12195$, 2015.

[8] SAYEGH, A.; MUNIR, S.; HABEEBULLAH, T. $M$. Comparing the Performance of Statistical Models for Predicting PM10 Concentrations. International journal of Aerosol and Air Quality Research, n. 14, p. 653-665, 2014.

[9] GRIVAS, G. ; CHALOULAKOU, A. Artificial neural network models for prediction of PM10 hourly concentrations, in the Greater Area of Athens, Greece. Atmospheric Environment, v. 40 , p. $1216-1229,2006$.

[10] SAWARAGI, Y. et al. Statistical Prediction of Air Pollution Le-vels Using Non-Physical Models. Automatica, v. 15, n. 4 p 441-451, 1979. DOI: https://doi.org/10.1016/0005-1098(79)90018-9.

[11] TADANO, Y. S. et al. Forecasting Particulate Matter Concentrations: Use of Unorganized Machines, International Journal of Advanced Engineering Research and Science, v. 4, p 188-191, Apr. 2017.

[12] MONTGOMERY, D; RUNGER, G. Applied statistics and probability for engineers. 3. ed. Arizona State University: Wiley, 2003.

[13] MATTOS NETO, P.S.G. de et al. An intelligent perturbative approach for the time series forecasting problem. In: IEEE WORLD CONGRESS ON COMPUTATIONAL INTELLIGENCE, 2010, Barcelona. Proceedings... Barcelona: IEEE, 2010. p.1-8.

[14] SOUZA, S. I. V. et al. Multiple linear regression and artificial neural networks based on principal components to predict ozone concentrations, Environmental Modelling \& Software, v. 22, p. $97-103,2007$.

[15] WILLMOTT, C. J. On the validation of models. Physical Geography, v. 2, p 184-194, 1981. 